\title{
METODOLOGÍA DE ANÁLISIS DE LIBROS DE TEXTO DE MATEMÁTICAS. LOS PUNTOS CRÍTICOS EN LA ENSEÑANZA SECUNDARIA EN ESPAÑA DURANTE EL SIGLO XX
}

\author{
González Astudillo, M. Teresa y Sierra Vázquez, Modesto \\ Departamento de Didáctica de la Matemática y de las Ciencias Experimentales. Universidad de Salamanca \\ maite@gugu.usal.es \\ mosiva@gugu.usal.es
}

\begin{abstract}
Resumen. Las investigaciones sobre libros de texto se están convirtiendo en un método eficiente para el estudio de los procesos de enseñanza aprendizaje. En este artículo se expone un instrumento para el análisis de dichos libros y se muestra cómo han evolucionado los conceptos relativos a los puntos críticos en los libros de textos españoles publicados a lo largo del siglo XX.
\end{abstract}

Palabras clave. Libros de texto, análisis matemático, puntos críticos.

Summary. The research on textbooks is becoming an efficient way to study the mathematics teaching and learning processes. In this paper is presented a new tool for textbook analysis and is shown the evolution of the concept of critical points in Spanish textbooks throughout the 20th century.

Keywords. Textbooks, mathematical analysis, critical points.

\section{INTRODUCCIÓN}

La implementación y utilización del libro de texto en el aula de matemáticas se ha producido de forma generalizada desde los inicios de la educación obligatoria hasta nuestros días, ejerciendo para ello diferentes papeles: como objeto de estudio, como material de consulta, como registro de las actividades del alumno, como colección de ejercicios propuestos y problemas a resolver... Esto ha originado una práctica escolar determinada por su uso, así como una organización de la enseñanza que se mantiene en la actualidad salvo casos aislados.
Desde el punto de vista histórico, en la transmisión del conocimiento, ha constituido un hito importante la aparición del libro escolar, que se puede considerar un elemento cultural reflejo de la manipulación social que selecciona unos contenidos frente a otros, que impone una determinada forma de estructurarlos y que propone a la siguiente generación cierto tipo de problemas con unas herramientas semióticas y no otras. En este sentido, Choppin (1980) considera que el libro de texto es «a la vez apoyo del saber en tanto que impone una distribu- 
ción y una jerarquía de los conocimientos y contribuye a forjar los andamios intelectuales tanto de alumnos como de profesores; es instrumento de poder, dado que contribuye a la uniformización linguiística de la disciplina, a la nivelación cultural y a la propagación de las ideas dominantes». Por ello, es interesante estudiar la contribución que los libros de texto han tenido en la historia de la educación matemática analizando la variedad y riqueza de sus contenidos, la incidencia en el aula, su función como transmisor de contenidos socialmente aceptados... Además, «los libros de texto determinan en la práctica la enseñanza más que los decretos de los distintos gobiernos». (Schubring, 1987)

Hay que destacar, además, que el papel de los libros de texto es doble e irreducible uno a otro (Otte, 1996): por un lado, su función comunicativa y de interpretación, que les dotará de un carácter subjetivo tanto desde el punto de vista del autor como del lector; y, por otro, su estructura materializada del conocimiento de un carácter eminentemente objetivo. Esta doble faceta de los libros de texto hace que su estudio aporte gran información tanto acerca de las concepciones en relación con el contenido matemático que desarrollan como acerca del proceso educativo con el que están relacionados.

En los últimos años, investigadores de diferentes ámbitos se han interesado por este tema, realizando algunos estudios de tipo general de forma que pudieran aplicarse a todas las áreas; pero son muy pocos los que se han ocupado del área de matemáticas específicamente. Los libros de texto constituyen una fuente de investigación para los interesados en la historia de la educación, ya que permiten estudiar los enfoques que se han dado, a lo largo de la historia, a una disciplina o concepto. Así, por ejemplo, en el proyecto MANES, que se inició en el año 1992 en España se fijó como objetivo fundamental realizar un estudio histórico de los manuales escolares publicados en nuestro país de 1808 a 1990: «[...] el proyecto tiene dos vertientes, una de carácter instrumental (histórico-documental), que requiere el censo de los manuales escolares editados en España a través de la elaboración de fichas bibliográficas que se recogen en una base de datos, así como otros tipos de documentos; y otra propiamente investigadora (histórico-educativa), que se está llevando a cabo mediante un conjunto de estudios y análisis historiográfico en torno a las características bibliométricas, editoriales, políticas, pedagógicas y curriculares de los libros de texto» (Tiana, 2000). Este proyecto se ha inspirado en otro homólogo que se venía desarrollando en el Institut National de Recherche Pédagogique de Francia desde 1980 bajo el nombre de Emmanuelle y con la dirección de Alain Choppin.

Entre los investigadores en educación matemática que han trabajado en torno al libro escolar, cabe destacar a Schubring (1987), que considera que el análisis de textos antiguos de matemáticas permite: extraer información sobre difusión y evolución de los saberes en una época determinada, interpretar fenómenos que tienen relación con los procesos de enseñanza-aprendizaje (representación, concepciones, aplicaciones), etc. En el marco de la investigación histórica en educación matemática, se ha puesto de manifiesto la importancia del análisis del libro de texto como reflejo de la actividad que se realiza en el aula. Así, Radford (1997) y Otte (1997) coinciden en considerar que es la actividad social la que determina el conocimiento y, según Fisher (1988), se puede decir que la matemática es «un constructo social que tiene sus raíces no sólo en el pensamiento individual, sino en las interacciones de las personas. Su conocimiento está aumentando gracias a su disponibilidad a través de libros, revistas, ordenadores...». Destaca, asimismo, el estudio comparativo de Howson realizado en 1995 sobre libros de texto de diferentes países. En este estudio además distingue entre investigaciones realizadas sobre textos $a$ posteriori, es decir, sobre la forma en que se ha usado un libro de texto, cómo ha contribuido al proceso de aprendizaje y qué obstáculos se han presentado, que son investigaciones más bien escasas; y las realizadas a priori, que son más numerosas. Entre las primeras destaca el estudio de Pepin y otros (2001) sobre la utilización de los libros de texto en Inglaterra, Francia y Alemania, en el cual concluyen que la forma de hacer matemáticas en las aulas depende en gran medida de un contexto más amplio en el que está inmersa dicha enseñanza y que es el que determina las formas culturales de un determinado país. Entre las segundas, hay que destacar los trabajos de Chevallard y sus colaboradores (Chevallard, 1985; Chevallard y Joshua, 1982), en los que se utiliza la noción de transposición didáctica relativa a las transformaciones entre el saber sabio y el saber enseñado y entre los que existe un escalón intermedio correspondiente al saber a enseñar que se refleja en el texto del saber. Este texto del saber es lo que el profesor piensa que tiene que enseñar una vez que se han publicado las orientaciones y libros de texto y se ha fijado la interpretación del currículo a través de diversos proyectos de centro y aula. Lo más próximo a este texto del saber, o saber a enseñar, es el libro de texto, cuyo contenido y estructura reflejan esas transformaciones del saber sabio.

Otras investigaciones se centran en aspectos relativos al lenguaje y la legibilidad de los textos (Pimm, 1987, 1994) o en la forma de presentación de los contenidos como la de Otte (1987), que pone el énfasis en lo que transmite el texto, las relaciones entre el conocimiento y la representación textual y las variaciones en las interpretaciones. A su vez, Dormolen (1986) hace una clasificación de los elementos que son imprescindibles en un libro de texto de matemáticas, y Lowe y Pimm (1996) consideran que hay una tétrada asociada a un libro de texto: el lector, el escritor, el profesor y el mismo libro, y que las características de cada uno de ellos, así como sus interacciones determinan el uso de este material en el aula. Resulta, a su vez, imprescindible destacar el trabajo realizado por Dhombres (1984) y el ya citado de Schubring (1987) sobre metodología de análisis histórico de libros de texto, en el que se resalta la necesidad de una aproximación global que analice los cambios en las sucesivas ediciones de un libro de texto, los cambios respecto a otros libros de texto y la relación de éstos con los que se han producido en el contexto. También hay que tener en cuenta los trabajos de Cantoral (1995), Filloy y 
Rojano (1984) y Puig (1994) para comparar algunos de los procesos utilizados por los alumnos en la comprensión del conocimiento matemático y los utilizados en los libros o textos históricos de matemáticas.

En España los trabajos realizados son más bien escasos, pero debemos destacar: el estudio de Sanz (1995) sobre los tipos y la función de las configuraciones gráficas de datos en los libros de texto de primaria; el de Maz (2000) acerca de la forma de presentar los números negativos en los textos de matemáticas de los siglos XVIII y XIX; los trabajos de Sierra, González y López (1999, 2003) sobre la evolución de los conceptos de límite y continuidad en los libros de texto de matemáticas de España; y la investigación de González (2002) sobre la evolución del concepto de punto crítico, desde los libros históricos de texto de los siglos XVII y XVIII hasta los libros de educación secundaria utilizados en España durante el siglo XX.

En este artículo se analiza la presentación que hacen los libros de texto de los contenidos relativos a los puntos críticos. Las razones por las que se decidió este tema, frente a otros contenidos del análisis matemático que también se incluyen en la enseñanza secundaria, son que «es un tópico que aparece en todos los libros de texto [...] es uno de los motores que dio pie al nacimiento del cálculo diferencial, una de las aplicaciones más características de éste y una fuente de problemas y fenómenos que podrían caracterizar las situaciones didácticas en los libros de texto». (González, 2002)

\section{METODOLOGÍA}

A lo largo de este trabajo se ha utilizado el término $l i$ bro de texto para designar aquellos libros que utilizan habitualmente profesores y alumnos a lo largo del curso escolar en el proceso de enseñanza-aprendizaje de un área de conocimiento. En la terminología francesa, se suele utilizar el término manuel scolaire y en la inglesa los términos textbook y schoolbook como sus equivalentes, mientras que se utiliza workbooks para designar los cuadernos de ejercicios del alumno. Su contenido es una estructura fácilmente reconocible, por la subdivisión en capítulos y temas que siguen los periodos básicos del calendario escolar.

El interés del análisis sobre libros de texto parte de la hipótesis de que la práctica de la enseñanza no está tan determinada por los decretos y órdenes ministeriales como por los libros de texto utilizados en el aula (Sierra, González y López, 1999). La producción de libros de texto se lleva a cabo dentro de un contexto determinado y responde a las corrientes epistemológicas y didácticas al uso. Tampoco debemos olvidar los condicionantes económicos como la rentabilidad del producto para el autor o para la editorial. Además, existiendo en el caso español disposiciones oficiales sobre el currículo, los libros de texto tienden a adaptarse a ellas. Por esta razón, se han agrupado los libros en periodos que, en líneas generales, corresponden a los sucesivos planes de estudio. Aunque el cálculo diferencial ya se había incluido en las enseñan- zas que los jesuitas establecieron en su Ratio Studiorum, concretamente en la versión del padre Pachtler, hemos iniciado este estudio a partir del plan de 1934, en el que por primera vez se incluye el análisis matemático en las enseñanzas regladas establecidas en España durante el siglo XX. Estos planes han sido agrupados en cuatro periodos teniendo en cuenta las orientaciones de cada uno de ellos y la similitud entre algunos:

- La introducción de la enseñanza del análisis matemático: 1934-1967. En este periodo se incluyen los planes correspondientes a los años 1934, 1938 y 1953.

- Introducción de la matemática moderna: 1967-1975. Este periodo abarca desde la introducción de la matemática moderna hasta la implantación del bachillerato unificado y polivalente (BUP) en 1975.

- Desarrollo del plan de estudios de BUP: 1975-1995. Este periodo comprende desde la implantación del BUP hasta el inicio de los nuevos bachilleratos derivados de la Ley de Ordenación General del Sistema Educativo (LOGSE).

- Una nueva orientación de la enseñanza de las matemáticas bajo las orientaciones de la LOGSE: 19952001. Corresponde al plan de estudios de 1992.

En un primer momento se realizó una selección de libros de texto teniendo en cuenta los autores «más» famosos o las editoriales más sobresalientes en cada uno de los periodos. Los libros correspondientes a cada uno de estos periodos aparecen en la tabla I.

Dada la magnitud de manuales encontrados y teniendo en cuenta investigaciones anteriores realizadas por este grupo investigador, se ha realizado una selección más afinada procurando que la pérdida de información en relación con la cantidad de libros sea mínima. En investigaciones anteriores establecimos que los libros de la lista anterior se pueden clasificar, dentro de cada uno de los periodos, en tres grupos según las orientaciones que dan a los conceptos de análisis matemático, por lo que se eligió un libro de cada uno de dichos grupos que representa a otros que son similares. Estos grupos vienen caracterizados por: $a$ ) según que sigan las orientaciones establecidas en el periodo anterior; $b$ ) que sean fieles a las orientaciones y normativas del periodo al que pertenece; y $c$ ) que sean precursores de nuevos métodos y formas de entender la enseñanza de las matemáticas. En definitiva, los libros a los que se ha realizado el análisis, clasificados según los periodos establecidos anteriormente, han sido los siguientes:

\section{- Los inicios de la enseñanza del análisis matemático: 1934-1967}

1) Baratech, B. y Royo Villanova (1938). Matemáticas. Séptimo curso de bachillerato. Primera parte. Análisis. Zaragoza: Librería General.

2) Fernández de Castro, M. y Jiménez Jiménez, J.L. (anterior a 1955). Matemáticas. Tercero, cuarto, quinto, sexto y séptimo. Preparación del examen de estado. Cádiz: Escalicer. 
Tabla I

Lista de libros seleccionados.

\begin{tabular}{|c|c|}
\hline Periodo & Libros \\
\hline Primer periodo & $\begin{array}{l}\text { Baratech, B. y Royo Villanova (1938). Matemáticas. Séptimo curso de bachillerato. Primera parte. Análisis. } \\
\text { Zaragoza: Librería General. } \\
\text { Carranza, E. (1972). Matemáticas. Sexto curso. Bachillerato. Plan 1957. Madrid: Summa. } \\
\text { Fernández de Castro y Jiménez Jiménez (anterior a 1955). Matemáticas. Preparación del examen de estado. Cádiz: Escelicer. } \\
\text { Bruño (1968). Matemáticas } 6^{\circ} \text { curso. Madrid: Bruño. } \\
\text { Sixto Ríos y Rodríguez San Juan (1950). Matemática. Sexto curso de bachillerato. Madrid: Los autores. } \\
\text { Sixto Ríos y Rodríguez San Juan (1968). Matemáticas. Sexto curso de bachillerato. Madrid: Los autores. } \\
\text { Sixto Ríos y Rodríguez San Juan (1968). Matemáticas. Quinto curso de bachillerato. Madrid: Los autores. }\end{array}$ \\
\hline Segundo periodo & $\begin{array}{l}\text { Abellanas y otros (1967). Matemática moderna. Quinto curso. Madrid: MEC. } \\
\text { Abellanas y otros (1969). Matemática moderna. Sexto curso. Madrid: MEC. } \\
\text { Sin autor (1972). Matemática sexto curso. Zaragoza: Edelvives. } \\
\text { Segura, S. (1974). Matemáticas } 6^{\circ} \text { curso. Valencia: ECIR. } \\
\text { Tudurí, J. y Casal, R. (1974). COU Matemáticas especiales. Tarrasa: Vimasa. } \\
\text { Marcos de Lanuza (1972). Matemáticas } 5^{\circ} \text { curso. Madrid: G. del Toro. } \\
\text { Marcos de Lanuza, F. (1972). Matemáticas COU optativo. Madrid: G. del Toro. } \\
\text { Agustí, J. y Vila, A. (1973). Matemáticas } 6^{\circ} \text { Bachillerato. Barcelona: Vicens-Vives. } \\
\text { Marcos, C. y Martínez, J. (1973). Matemáticas generales COU. Valencia: SM. }\end{array}$ \\
\hline Tercer periodo & $\begin{array}{l}\text { Marcos de Lanuza, F. (1978). Matemáticas } 2^{\circ} \text { BUP. Madrid: G. del Toro. } \\
\text { Marcos de Lanuza, F. (1976). Matemáticas especiales. COU. Madrid: G. del Toro. } \\
\text { Agustí, J. y Vila, A. (1976). Matemáticas. Vectores } 2^{\circ} \text { BUP. Barcelona: Vicens-Vives. } \\
\text { Etayo, J. y otros (1977). Matemáticas } 2^{\circ} \text { de BUP. Salamanca: Anaya. } \\
\text { Guillén, J. y otros (1976). Matemáticas } 2^{\circ} \text { bachillerato. Madrid: Magisterio. } \\
\text { Boadas, J. y otros (1977). Matemáticas } 2^{\circ} \text { de bachillerato. Barcelona: Teide. } \\
\text { Anzola, M. y otros (1976). Matemáticas } 2^{\circ} \text { bachillerato. Madrid: Santillana. } \\
\text { Grupo Cero (1985). Matemáticas de bachillerato. Barcelona: Teide. } \\
\text { Guzmán, M. y Colera, J. (1988). Matemáticas. Bachillerato 3. Madrid: Anaya. }\end{array}$ \\
\hline Cuarto periodo & $\begin{array}{l}\text { Lazcano, I. y Barolo, P. (1993). Matemáticas } 2^{\circ} \text { BUP. Madrid: Edelvives. } \\
\text { Álvarez, F. y otros (1992). Matemáticas. Factor 2. Barcelona:Vicens-Vives } \\
\text { Hernández, F. y otros (1989). Matemáticas } 2^{\circ} \text { bachillerato. Bruño: Madrid. } \\
\text { Colera, J. y Guzmán, M. (1994). Bachillerato. Matemáticas 2. Madrid: Anaya. } \\
\text { Guzmán, J. y Colera, J. (1989). Matemáticas I COU. Madrid: Anaya } \\
\text { Primo, A. y otros (1998). Matemáticas (Modalidad: tecnología, ciencias de la naturaleza y de la salud). } \\
\text { Salamanca: Hespérides. } \\
\text { Vizmanos, R. y Anzola, M. (1998). Matemáticas } 2 \text { (Ciencias de la naturaleza y de la salud - tecnología). Madrid: SM. } \\
\text { Biosca, A. y otros (1999). Matemáticas II. Barcelona: Edebé. }\end{array}$ \\
\hline
\end{tabular}

3) Carranza, E. (1972). Matemáticas. Sexto curso. Bachillerato. Plan 1957. Madrid: Summa.

- Introducción de la matemática moderna: 1967-1975

1) Sin autor (1972). Matemáticas. Sexto curso. Zaragoza: Luis Vives.

2) Abellanas, P. y otros (1969). Matemática moderna. Sexto curso. Madrid: MEC.

3) Marcos, C. y Martínez, J. (1973). Matemáticas especiales. COU. Madrid: SM.

- Desarrollo del plan de estudios de BUP: 1975-1995

1) Marcos de Lanuza, F. (1978). Matemáticas $3^{\circ}$ BUP. Madrid: G. del Toro.

2) Etayo, J. y otros (1977). Matemáticas $3^{\circ}$ BUP. Salamanca: Anaya.
3) Grupo Cero (1982). Matemáticas de bachillerato, volumen 2. Barcelona: Teide.

4) Guzmán, M., Colera, J. y Salvador, A. (1988). Matemáticas. Bachillerato 3. Madrid: Anaya.

- Una nueva orientación de la enseñanza de las matemáticas bajo las orientaciones de la LOGSE: 19952001.

1) Primo, A. y otros (1998). Matemáticas (Modalidad: tecnología, ciencias de la naturaleza y de la salud). Salamanca: Hespérides.

2) Vizmanos, R. y Anzola, M. (1998). Matemáticas 2 (Ciencias de la naturaleza y de la salud-tecnología). Madrid: SM.

3) Biosca, A. y otros (1999). Matemáticas II. Barcelona: Edebé. 
Aunque el estudio se ha llevado a cabo de forma independiente en cada uno de los periodos, la estructura que se ha seguido para cada uno de ellos es similar, realizándose en tres etapas, cada una de las cuales profundiza el trabajo realizado en la etapa anterior.

En primer lugar se ha intentado contextualizar cada uno de los periodos, exponiéndose las orientaciones oficiales que se establecieron en ellos, para poder analizar los libros a la luz de dichas consideraciones. Fundamentalmente se han utilizado los programas oficiales elaborados por el Ministerio de Educación para poder completar esta fase.

Posteriormente se hace un análisis de la forma de presentación de los puntos críticos en cada uno de los libros, estudiando las formas de expresión matemática que en ellos se incluyen. Se ha de tener en cuenta que la forma en que se expresan los conceptos matemáticos, no sólo en los libros de texto sino en prácticamente cualquier medio y bajo cualquier soporte es muy variada. Se considera que los principales modos de representación (Janvier, 1987) son cuatro: descripciones verbales, tablas de datos, representaciones gráficas y expresiones simbólicas. Para cada una de estas formas de representación se han ido seleccionando sucesivas unidades de información procedentes de los textos estudiados, sobre las que se ha procedido a realizar el análisis. Asimismo, como según Rojano (1994) y Palarea (1999), «se detecta, sin embargo, la ausencia de un paradigma para el estudio del sistema matemático de signos, que abarque sus aspectos sintáctico, semántico, pragmático y sociocultural», se realizó un análisis basado en las siguientes categorías:

- Sintáctico, ya que cada símbolo se puede considerar susceptible de ser insertado en secuencias junto con otros símbolos mediante unas reglas que garantizan la coherencia interna y la validez.

- Semántico, puesto que los signos se consideran en relación con su significado matemático y sus relaciones con conceptos de otras ciencias.

- Pragmático-didáctico, siempre que el signo se considere en relación con la utilización que se hace de él desde el punto de vista didáctico para, por ejemplo, resaltar unas características del concepto que representa frente a otras. En este sentido, una representación puede ser más adecuada que otra, en función del uso que se vaya a hacer de ella. La utilización puede indicarnos si son representaciones sólo de lectura, si hay que completar, si tenemos una fuente de datos, un ejemplo, prototipo o ilustración de algo, o bien si están integradas en una actividad. Incluso tendremos que tener en cuenta algunos aspectos que pueden estar relacionados con las herramientas que se están utilizando en un determinado momento; por ejemplo, cuando hacemos gráficas con calculadoras gráficas, hemos de tener en cuenta: la escala usada, la ventana definida en la calculadora...

- Sociocultural, que hace referencia a símbolos, términos y cualidades de estos símbolos, y términos característicos de una determinada época o de una determinada cultura o sociedad. Muchos de los símbolos matemáticos son intemporales, desde el momento de su introducción no ha variado su uso. Actualmente, con la introducción de las nuevas tecnologías, veremos cómo las necesidades tecnológicas han impuesto algunas variaciones en estos símbolos.

Se ha elaborado un instrumento para el análisis, definiéndose veinte dimensiones agrupadas en las cuatro categorías anteriores. En cada dimensión se han considerado tres modalidades. Esto ha permitido clasificar los manuales en tres perfiles según la modalidad dominante:

- Expositivo (E). Son libros en los que se considera el conocimiento matemático como una acumulación de enunciados, reglas y procedimientos aislados, y relativamente inconexos y desconectados de la realidad, pero que poseen una estructura matemática, típicamente deductiva, en la que, partiendo de las definiciones de los conceptos, se deducen los teoremas y se exponen algunos pocos ejemplos: es una estructura ciertamente prescriptiva. Esta estructura implica, en cuanto a la enseñanza, que los objetivos son conceptuales: incita a la exposición magistral y a la ejercitación repetitiva. Este tipo de libros induce a un aprendizaje de tipo memorístico, en los que importa más la estructura matemática que la comprensión de los conceptos, a pesar del énfasis que se pone en las definiciones y teoremas.

- Tecnológico (T). Se conciben las matemáticas como una organización lógica de enunciados, reglas y procedimientos que se emplean como técnicas o destrezas para pensar sobre los conceptos y aplicarlos a diversas situaciones. Las distintas ramas de las matemáticas aparecen totalmente desconectadas. A partir de objetivos terminales u operativos, y por medio de una estructura secuencial en la enseñanza, se intenta una ejercitación productiva, proponiéndose para ello numerosas aplicaciones con la intención de dotar de sentido a las distintas reglas. Aunque los procedimientos y conceptos están organizados y estructurados de una forma lógica, se hace más énfasis en la memorización de reglas y la aplicación en ejercicios y problemas.

- Comprensivo (C). Se conciben las matemáticas como un instrumento para interpretar la realidad entendida ésta en sentido amplio. En este caso se parte de objetivos flexibles, de forma que para conseguirlos se requiere la experimentación, por lo que el tipo de enseñanza adecuada es la realizada por descubrimiento, permitiendo de esta forma la construcción de redes conceptuales. El aprendizaje de las matemáticas se adquiere mediante el establecimiento de una red de relaciones con otros contenidos que pueden ser matemáticos o no, dando así sentido a las matemáticas. Se considera que los conceptos se adquieren partiendo de situaciones propias de la realidad que permiten la construcción de conceptos y reglas.

En la tabla II se presentan de manera sintética las categorías y las dimensiones utilizadas (Anexo). Para cada categoría se consideran cinco dimensiones, una por cada uno de los sistemas de representación: descripciones verbales (distinguiendo entre problemas y definiciones), tablas de datos, representaciones gráficas y expresiones simbólicas. La lectura horizontal permite comparar las tendencias de los diferentes tipos de texto. La lectura vertical permite identificar las diferentes dimensiones que se han tenido en cuenta, lo que da una visión global del tipo de análisis realizado. 
Tabla II

Categorías, dimensiones y perfiles.

\begin{tabular}{|c|c|c|c|c|c|}
\hline Categorías & & Dimensiones & Expositivo & Tecnológico & Comprensivo \\
\hline \multirow{5}{*}{ Sintáctica } & 1 & Estructura del problema & Clásica & Aplicación & Explicación \\
\hline & 2 & Descripciones teóricas & Formales & Formales-intuitivas & Intuitivas \\
\hline & 3 & Símbolos utilizados en las tablas & Sin tablas & Con símbolos matemáticos & Con iconos \\
\hline & 4 & Símbolos utilizados en las gráficas & Literal & Utilización de números & Elementos explicativos \\
\hline & 5 & Tipos de expresiones simbólicas & Familias & Específicas & Variadas \\
\hline \multirow{5}{*}{ Semántica } & 6 & Fenomenología & Matemáticas & Realistas & Reales \\
\hline & 7 & Tipos de descripciones & De conceptos & De reglas & De relaciones \\
\hline & 8 & Tipos de tablas & Sin tablas & Descripción local & Cuadros de variación \\
\hline & 9 & Tipos de gráficas & Ideogramas & Ábacos & Mensajes topológicos \\
\hline & 10 & $\begin{array}{l}\text { Significado de las expresiones } \\
\text { simbólicas }\end{array}$ & Objeto & Regla & Proceso \\
\hline \multirow{5}{*}{$\begin{array}{l}\text { Pragmático- } \\
\text { didáctica }\end{array}$} & 11 & Función de los ejercicios & Rutinarios & Aplicación & Deducción \\
\hline & 12 & Papel de las definiciones & $\begin{array}{l}\text { Estructurales- } \\
\text { teóricas }\end{array}$ & Aplicación a problemas & Interpretación \\
\hline & 13 & Actividades relacionadas con las tablas & Sin tablas & Construcción & Interpretación/Construcción \\
\hline & 14 & Actividades gráficas & Visualización & Construcción & Interpretación/Construcción \\
\hline & 15 & Papel de las expresiones simbólicas & Ejemplificación & Escolar & Social \\
\hline \multirow{5}{*}{$\begin{array}{l}\text { Socio- } \\
\text { cultural }\end{array}$} & 16 & $\begin{array}{l}\text { Influencia social y adaptación al } \\
\text { currículo }\end{array}$ & No hay & Contexto intemporal & Contexto actual \\
\hline & 17 & Influencias didácticas & Clásica & Adaptada al currículo & Novedosa \\
\hline & 18 & Aplicación de las tablas & Sin tablas & Elemento auxiliar & Categoría de objeto \\
\hline & 19 & $\begin{array}{l}\text { Presentación de las gráficas (estática/ } \\
\text { dinámica) }\end{array}$ & Descontextualizada & Impresa & Nuevas tecnologías \\
\hline & 20 & $\begin{array}{l}\text { Complejidad de las expresiones } \\
\text { simbólicas }\end{array}$ & Clásicas & Sencillas & Complejas \\
\hline
\end{tabular}

Para obtener el perfil de cada libro se ha partido de las unidades de información seleccionadas en cada uno de ellos, asignándoles una tétrada de dimensiones (una para cada categoría: sintáctica, semántica, pragmática y didáctica). Posteriormente, se han elaborado tablas resumen para cada periodo y cada libro, que permiten visualizar y comprobar la prevalencia de las diferentes orientaciones en cada una de las categorías y dimensiones. Y finalmente, se ha completado la tabla anterior para cada libro, indicando, respecto a cada dimensión mediante un asterisco en la casilla correspondiente, la orientación que se ha obtenido del análisis del libro. Para una misma dimensión, puede haber varios asteriscos, en el caso de que haya unidades de información que correspondan a varios perfiles, y la diferencia en la cantidad de las unidades correspondiente a cada uno de ellos sea pequeña. El libro quedará clasificado en la columna en la que aparezca mayor número de asteriscos.

\section{RESULTADOS}

En este apartado se exponen los resultados que se han obtenido en relación con cada uno de los periodos en los que se ha dividido la enseñanza del análisis matemático durante el siglo XX en España, siguiendo la metodología descrita en el apartado anterior. La estructura de cada uno de los periodos es similar, iniciándose con un breve resumen correspondiente a los planes de estudio y orientaciones oficiales del periodo en cuestión (para una visión más pormenorizada de los planes de estudio, ver Sierra, González y López, 1999, 2002). Se continúan explicitando las características de los libros de texto del periodo concretado en relación con los puntos críticos cuáles son las representaciones más habituales y la función que cumplen en el texto; y, finalmente, se presenta, con más detalle, el perfil de uno de los libros analizados como ejemplo de una de las versiones en las que se han concretado las características anteriores. En dicho perfil se describen inicialmente algunas de las características que identifican al libro de texto en cuestión y que se recogen en la tabla de perfil a modo de esquema y después de cada tabla se hace un pequeño resumen indicando cuál es el perfil del libro elegido y por qué se ha asignado dicho perfil.

\subsection{Los inicios de la enseñanza del análisis mate- mático (1934-1967)}

\section{Planes de estudio}

En este periodo estarán vigentes los planes de estudio de 1934, 1938 y 1953 con las modificaciones de 1957 que 
estructuran el bachillerato en siete años, incluyendo por primera vez contenidos de análisis matemático en los dos últimos cursos. Inicialmente los cursos eran comunes sin hacer distinción entre especialidades, pero posteriormente se inicia una división en dos especialidades denominadas Ciencias y Letras.

\section{Características de los libros de texto}

Al ser éste el primer periodo en la enseñanza del análisis matemático en la educación secundaria en España, no tenemos ningún otro modelo con el que podamos compararlo, por lo tanto, marcará la pauta de comparación del resto de los periodos. Introducir los conceptos de esta rama de las matemáticas en la enseñanza secundaria constituye un hito en cuanto a la consideración que los nuevos programas van a tener en relación con ellos, de tal forma que se considera necesaria la incorporación de conceptos de matemáticas superiores en la preparación de los estudiantes que posteriormente ingresarán en las universidades, sobre todo en las carreras técnicas, y que necesitarán este tipo de conceptos. Se incluyen en los últimos cursos de bachillerato, junto con otras ramas de las matemáticas, como la geometría analítica y los números complejos, siendo su carácter fundamentalmente formativo y son de carácter obligatorio para todos los alumnos, destacando así la importancia que se les concede.

La estructura de los libros evoluciona a lo largo de este periodo, desde el de Fernández y Jiménez, de tipo enciclopédico, hasta los libros de Baratech y Royo, y Carranza, con una organización para un solo curso, dividida en lecciones, que es más actual y en los que al final de cada capítulo se incluye una colección de ejercicios o problemas para practicar los conocimientos adquiridos a lo largo de cada lección de forma similar a los libros actuales.

En estos libros también se observa una evolución en el lenguaje matemático que todavía no es el definitivo, en Fernández y Jiménez no se considera el concepto de función, sino que se desarrolla todo el análisis matemático a partir del concepto de variable, y se manejan términos típicos de este lenguaje como el relativo a los infinitésimos. Poco a poco, a medida que va avanzando el periodo, el concepto de función va adquiriendo un papel que culminará en el siguiente periodo.

Los fenómenos seleccionados para trabajar los puntos críticos son típicos del periodo, destacando, en comparación con los demás, la inclusión de numerosos problemas y ejercicios relacionados con conceptos propios de física, como la luminosidad, la elasticidad, la velocidad... y con herramientas, como palancas. También forman un capítulo importante los problemas de carácter geométrico, dada la entidad que tenía la geometría en la enseñanza en la época que nos ocupa en relación con otras épocas más actuales. Muchos problemas planteados son de tipo genérico asociados a toda una familia de funciones, heredando la forma de hacer de los matemáticos clásicos.

En cuanto a las definiciones y descripciones más teóricas, destaca la incorporación de algunos símbolos matemáticos, como los relativos a las funciones, los símbolos relacionales $>,<$, algunas letras griegas como $\varepsilon, \Delta$, la utilización de subíndices y superíndices... Dichas definiciones descansan en el aspecto gráfico de las funciones, siendo continuas las referencias a la curva que representa gráficamente la función. Las tablas son muy escasas y su uso se reduce a ser meros auxiliares para la representación gráfica de las funciones. Las gráficas también son escasas; mediante una única gráfica se representan todas las posibilidades en relación con los puntos críticos, presentándose no sólo los casos que se corresponden con las funciones derivables, sino también aquéllas que no lo son. Las expresiones simbólicas son casi todas algebraicas, siendo las más numerosas las polinómicas. En algunas expresiones se utilizan en lugar de los símbolos $x$ e $y$ para las variables, $e$ y $t$, y se destaca la importancia y la aplicación de los conceptos del análisis matemático en relación con la física.

\section{Perfil}

Entre los libros que se han analizado presentamos a continuación los resultados correspondientes a uno de ellos: Emilio Pérez Carranza (1972). Matemáticas. Sexto curso. Bachillerato. Plan 1957. Madrid: Editorial Summa.

Este libro utiliza el lenguaje de funciones de forma explícita, sin hacer referencia a las variables, con lo cual el concepto de función va adquiriendo cierto estatus que no poseía en los libros de texto hasta este momento. El autor se limita a definir los conceptos y apenas hay demostraciones, sino simples comprobaciones; a pesar de ello, tanto las definiciones como las reglas de cálculo o los algoritmos son guiados durante la exposición de la lección, obteniéndose de una forma casi deductiva. La noción de matemática que se muestra es de una matemática ya hecha, que sólo hay que memorizar. La estructura de los enunciados es la típica utilizada en la formulación matemática, tanto en relación con los problemas como con las definiciones y las reglas de cálculo:

Encontrar el número $x$ cuya raíz de índice $x$ es máxima. (Carranza, p. 124)

De todos los rectángulos isoperimétricos, calcular el de área máxima. (Carranza, p. 121)

Como muchos problemas son de tipo general, las soluciones que se obtienen también lo son, por lo que no importa tanto la forma que tienen las soluciones como su significado. Así se dice: «[...] de todos los rectángulos isoperimétricos, el cuadrado es el de área máxima». De esta forma se ofrece una interpretación cualitativa de los resultados obtenidos. De todas formas también hay ejercicios que hacen referencia a otras ciencias como la física:

Se supone que un plano está descompuesto por una recta en dos semiplanos, que son dos medios homogéneos en los que las velocidades respectivas de la luz son $v_{1} \mathrm{y} v_{2}$.

Encontrar el camino que debe seguir un rayo luminoso para ir en el menor tiempo posible (camino óptico) desde un punto A, situado en uno de los medios, a otro B, situado en el otro medio. (Carranza, p.121) 
O situaciones de tipo real que reflejan el contexto social de la época:

Un barco movido con aceite pesado consume por hora un número de litros igual al cubo de su velocidad en nudos. Tal combustible cuesta a 5 ptas. el litro; los demás gastos del barco son 25.000 pesetas por hora. Calcular la velocidad del barco para reducir a un mínimo los gastos de un recorrido de 1.000 millas. (Carranza, p. 123)

Las definiciones hacen referencia a la gráfica de la función y, por lo tanto, a la posición geométrica de los puntos críticos en relación con el resto de puntos de la gráfica.

Se dice que una función $y=f(x)$ admite un máximo relativo para un valor de $x$ cuando es creciente a su izquierda y decreciente a su derecha. (Carranza, p. 119)

En este libro no hay ninguna tabla, y las gráficas son ideogramas en las que se presentan todos los diferentes tipos de puntos críticos: un máximo, un mínimo y dos puntos de inflexión. La utilidad de estas gráficas viene determinada porque sirve de ilustración, boceto o ejemplo del tipo de comportamiento de la gráfica de una función en estos puntos. Mediante el trazado de las rectas tangentes, se señala la relación que existe entre la tangente en un punto y la gráfica. Se utilizan las líneas punteadas como recurso para relacionar la abscisa con el punto de la curva que tiene tangente horizontal. (Carranza, p. 118)

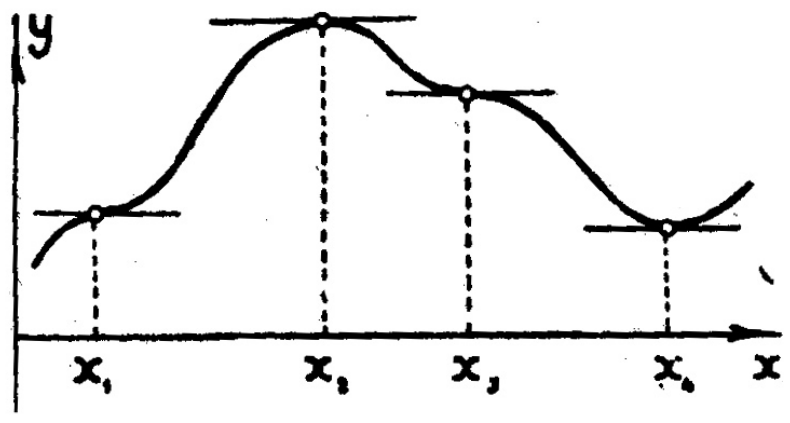

En cuanto al tipo de funciones utilizadas hay una gran variedad, desde las más sencillas polinómicas hasta otras de tipo racional, trascendente, y una que contiene un valor absoluto.

El libro de Carranza es un libro expositivo, en el que se pone el interés en el desarrollo conceptual del tema y se establece un cierto desarrollo de las reglas y técnicas necesarias para el cálculo de los puntos críticos. Semánticamente se utilizan ciertas representaciones que tratan de dotar de significado a los conceptos y de abarcar diferentes posibilidades. Hay algunas referencias a la sociedad del momento y se valoran algunos aspectos comprensivos. El libro es un fiel reflejo de la necesidad de dotar de coherencia y de reflejar la estructura que posee la matemática propia de aquella época (Tabla III).

Tabla III

Perfil del libro de Carranza.

\begin{tabular}{|c|c|c|c|c|}
\hline Categorías & Dimensiones & Expositivo & Tecnológico & Comprensivo \\
\hline \multirow{5}{*}{ Sintáctica } & Estructura del problema & $*$ & & \\
\hline & Descripciones teóricas & $*$ & $*$ & \\
\hline & Símbolos utilizados en las tablas & $*$ & & \\
\hline & Símbolos utilizados en las gráficas & $*$ & & $*$ \\
\hline & Tipos de expresiones simbólicas & & $*$ & \\
\hline \multirow{5}{*}{ Semántica } & Fenomenología & $*$ & & \\
\hline & Tipos de descripciones & & $*$ & $*$ \\
\hline & Tipos de tablas & $*$ & & \\
\hline & Tipos de gráficas & & & $*$ \\
\hline & Tipos de expresiones simbólicas & & * & $*$ \\
\hline \multirow{5}{*}{ Pragmático-didáctica } & Función de los ejercicios & $*$ & & \\
\hline & Papel de las definiciones & * & * & \\
\hline & Actividades relacionadas con las tablas & $*$ & & \\
\hline & Actividades gráficas & & & $*$ \\
\hline & Papel de las expresiones simbólicas & & * & \\
\hline \multirow{5}{*}{ Sociocultural } & Influencia social y adaptación al currículo & $*$ & & \\
\hline & Influencias didácticas & $*$ & & \\
\hline & Aplicación de las tablas & $*$ & & \\
\hline & Presentación de las gráficas & $*$ & & \\
\hline & Complejidad de las expresiones simbólicas & & $*$ & \\
\hline
\end{tabular}




\subsection{Introducción de la matemática moderna: 1967-1975}

\section{Planes de estudio}

Este periodo abarca desde la introducción de la matemática moderna hasta la implantación del bachillerato unificado y polivalente (BUP) en el año 1975. Durante la década de los sesenta, la «matemática moderna» se va introduciendo en los programas de bachillerato. En 1962, se constituyó la Comisión para el Ensayo Didáctico sobre Matemática Moderna en los Institutos Nacionales (creada por OM de 7 de diciembre de 1961) presidida por el profesor Abellanas, que editó, en los años 1967 y 1969, textos piloto para los cursos $5^{\circ}$ y $6^{\circ}$ de bachillerato respectivamente. El Ministerio además editó unos cuadernos didácticos dedicados a desarrollar temas de matemáticas modernas y desde el año 1963 hasta el año 1966, la revista Enseñanza Media publicó una serie de artículos bajo el título «Lecciones de matemática moderna»; de modo que, progresivamente, se fue implantando un programa en el bachillerato, cuyos cimientos son la teoría de conjuntos y que maneja las estructuras matemáticas en sentido bourbakista, es decir, las estructuras algebraicas, de orden y topológicas. De hecho, los textos piloto se convirtieron en el nuevo programa de matemáticas en el bachillerato.

\section{Características de los libros}

Este periodo, como hemos indicado, está marcado por la introducción de la matemática moderna, que, en lo que se refiere al análisis, introducirá el lenguaje y la estructura típica de la topología, por lo que son habituales términos como entorno, máximo (mínimo), relativo (absoluto), así como el empeño en la exactitud en el lenguaje y en la estructura axiomática de los temas. El concepto de función tiene entidad propia a lo largo de este periodo, de forma que es un objeto de estudio y, dada la orientación de los programas de este periodo, se les dota de una estructura algebraica.

La estructura de los libros es la heredada de la época anterior, en la que los cursos se dividen en lecciones dedicadas a cada uno de los conceptos matemáticos desarrollados. La más novedosa es la del libro de SM, que resulta precursora respecto del siguiente periodo, ya que dotó a los libros de un carácter más escolar-didáctico, incluyendo explicaciones exhaustivas de cada uno de los conceptos que se describen. El libro de Edelvives continúa incluyendo problemas relativos tanto a la física como a la geometría, tan característicos de la época anterior, pero los otros libros plantean situaciones bastante más abstractas, de forma que hasta los problemas sobre situaciones cotidianas son adaptaciones hechas de problemas típicamente geométricos, para los que se ha buscado un contexto que sirviera de pretexto. Existe una ausencia importante en cuanto a los problemas que plantean situaciones generales, siendo más habituales los problemas numéricos específicos.

Las definiciones de los puntos críticos están más asociadas al valor de la función en un punto que a una determinada posición en su gráfica, y las reglas para su cálculo son de tipo algorítmico, estableciéndose pasos sucesivos algunos de los cuales equivalen a la resolución de una ecuación algebraica. La influencia de la matemática moderna tanto en las definiciones como en las reglas es clara. Las tablas, como en el periodo anterior, son escasas y ligadas a la representación gráfica de funciones. Destaca que no haya ninguna en el texto piloto y que, en las incluidas en el libro de SM, se empiece a incorporar algunos símbolos extramatemáticos como flechas. En este periodo se empiezan a utilizar las gráficas en forma de mensajes topológicos, en consonancia con el tipo de matemáticas que se están desarrollando y la importancia concedida a la topología. Las expresiones simbólicas son casi todas algebraicas. Se observa una gran ausencia de funciones no derivables, funciones definidas a trozos, y funciones de tipo más general que sí estaban incluidas en los libros del periodo anterior.

\section{Perfil}

Entre los libros que se han analizado se presenta el perfil de Abellanas, P. y otros (1969). Matemática moderna. Sexto curso. Madrid: MEC.

El texto piloto se puede considerar un libro clásico, fundamentalmente teórico, en el que la fenomenología es totalmente matemática: la matemática se encierra en sí misma y se explica por sí misma, incluso los diferentes conceptos matemáticos aparecen clasificados en distintas áreas de forma que no se relacionan unas con otras, manteniéndolas totalmente separadas. Los términos usados son formales, no dejando lugar a la intuición, la estructura de los enunciados es rigurosa y utiliza para ello tanto símbolos puramente matemáticos como locuciones y expresiones pertenecientes a la lógica matemática. Así, para definir lo que se entiende por un máximo relativo, se indica:

Se dice que la función $y=f(x)$ tiene un máximo relativo en el punto $x=a$ cuando

$$
f(a \pm h) \leq f(a)
$$

para $h$ suficientemente pequeño. (TP, p. 148)

Se hace hincapié en las técnicas que debería aprender el alumno de forma teórica. Así se indica cómo distinguir diferentes tipos de puntos de una función:

Sea $y=f(x)$ una función cuya derivada se anula en $x=a$. Supondremos que en cierto entorno de $a(a-h, a+h)$, y para todo valor de $x$ distinto de $a$, las derivadas $f^{\prime}(a)$ y $f^{\prime \prime}(a)$ son distintas de cero.

- Resulta, por tanto:

1) $f$ " $(a)<0$. La función presenta un máximo en $x=a$.

2) $f^{\prime \prime}(a)>0$. La función presenta un mínimo en $x=a$.

Si $f$ " $(a)=0$. hay que recurrir a derivados de orden superior. (TP, $\mathrm{p}$. $152-153)$

Apenas aparecen en él ejemplos, ejercicios y problemas, por lo que no hay ninguna referencia a elementos de la sociedad del momento. Además, en los problemas, las interpretaciones y las soluciones no se centran tanto en los aspectos cuantitativos como en relacionar dichos resultados con la teoría presentada. Al ser la presentación de los conceptos estática (como se puede observar en la definición de máximo relativo), los ejemplos están pensados totalmente para la práctica en el aula de los 
conceptos desarrollados inmediatamente antes. Así, en el siguiente ejemplo, el alumno ha de decidir que quiere conseguir un área máxima y aplicar los conocimientos recién adquiridos.

Con una cuerda de $2.000 \mathrm{~m}$ de longitud hemos de cercar un terreno que nos regalan y que debe tener forma rectangular. ¿Cómo hemos de colocar la cuerda? (TP, p. 157)

Los tipos de funciones son poco variados, la mayoría son funciones generales que se indican simplemente como $f(x)$, y los escasos ejemplos que se exponen son todos expresiones polinómicas, es decir, puramente algebraicas. No hay ningún tipo de interacción entre diferentes representaciones o sistemas matemáticos de signos, apareciendo, por lo tanto, cada uno de ellos de forma aislada y sin conexión, con lo que pierden bastante de su significado y de las interacciones que pudieran surgir entre ellas. De hecho, no aparece ninguna tabla de valores en relación con el tema que nos ocupa.

La representación algebraica de las funciones elegidas en los ejemplos está pensada en función de los cálculos que se hacen a posteriori.

En cuanto a la forma de presentación de las diferentes unidades de información solamente hay un uso del color azul para resaltar las representaciones gráficas, distinguir los diferentes apartados y los diferentes dibujos incluidos. Estas formas de representación se utilizan para identificar la posición de los puntos críticos en las gráficas a modo de esquema. En el siguiente ejemplo se muestra cómo aparecen representados de forma general los puntos máximos y mínimos. (TP, p. 149)
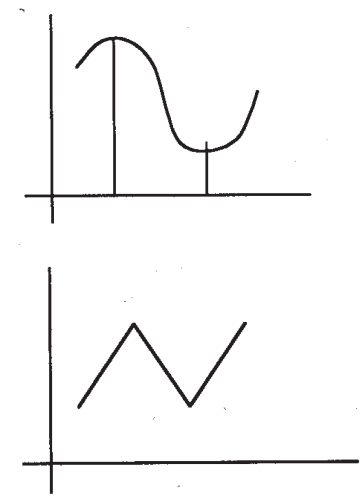

El texto piloto, como ya hemos comentado, es un libro de carácter expositivo en el que destacan el tipo de definiciones características de la matemática moderna y la ausencia de ejercicios y problemas para practicar los conceptos o reglas. A pesar de ello, dado el tipo de concepto que hemos estado analizando, los puntos críticos, cuya inclusión en los libros de texto está fundamentada en la aplicación del concepto de derivada a diversas situaciones, hacen que el tipo de definiciones y expresiones utilizadas en el libro estén enfocadas hacia la aplicación del concepto y por esto se puede observar en la tabla anterior que este perfil tiene también un carácter tecnológico. Los contextos son los grandes ausentes de este libro, se trata de desarrollar la matemática en sí misma (Tabla IV).

Tabla IV

Perfil del texto piloto.

\begin{tabular}{|c|c|c|c|c|}
\hline Categorías & Dimensiones & Expositivo & Tecnológico & Comprensivo \\
\hline \multirow{5}{*}{ Sintáctica } & Estructura del problema & & $*$ & \\
\hline & Descripciones teóricas & $*$ & $*$ & \\
\hline & Símbolos utilizados en las tablas & $*$ & & \\
\hline & Símbolos utilizados en las gráficas & $*$ & & $*$ \\
\hline & Tipos de expresiones simbólicas & & $*$ & \\
\hline \multirow{5}{*}{ Semántica } & Fenomenología & & $*$ & \\
\hline & Tipos de descripciones & & $*$ & $*$ \\
\hline & Tipos de tablas & $*$ & & \\
\hline & Tipos de gráficas & & & $*$ \\
\hline & Tipos de expresiones simbólicas & & $*$ & \\
\hline \multirow{5}{*}{ Pragmático-didáctica } & Función de los ejercicios & * & & \\
\hline & Papel de las definiciones & $*$ & $*$ & \\
\hline & Actividades relacionadas con las tablas & $*$ & & \\
\hline & Actividades gráficas & $*$ & & $*$ \\
\hline & Papel de las expresiones simbólicas & & $*$ & \\
\hline \multirow{5}{*}{ Sociocultural } & Influencia social y adaptación al currículo & & $*$ & \\
\hline & Influencias didácticas & $*$ & $*$ & \\
\hline & Aplicación de las tablas & $*$ & & \\
\hline & Presentación de las gráficas & $*$ & & \\
\hline & Complejidad de las expresiones simbólicas & & $*$ & \\
\hline
\end{tabular}




\subsection{Desarrollo del plan de estudios del bachillerato unificado y polivalente (BUP): 1975-1995}

\section{Planes de estudio}

A comienzos de la década de los setenta, se emprende en España una reestructuración del sistema educativo, que culmina con la aprobación de la Ley General de Educación y Financiamiento de la Reforma Educativa, promulgada el 4 de agosto de 1970. Los estudios debachillerato corresponden a los 14-17 años, las matemáticas están incluidas en el área de ciencias matemáticas y de la salud, y son obligatorias inicialmente en los tres cursos, aunque posteriormente, las de tercero pasaron a ser optativas. Durante este periodo el concepto del análisis matemático se incorpora a cursos inferiores respecto de los periodos anteriores, ya que se considera que su introducción ha de ser más progresiva, debe empezar antes e ir de forma cíclica completándose los contenidos. Se mantiene la herencia del periodo anterior, de forma que se puede decir que la matemática moderna sigue prevaleciendo a lo largo de la mayor parte de este periodo, salvo casos aislados como el libro del Grupo Cero y los últimos años, en los que los libros incorporan ciertos elementos novedosos como: un intento de aplicación de la llamada corriente de «resolución de problemas», la inclusión de notas históricas, anécdotas, curiosidades matemáticas, problemas de ingenio y entretenimiento, numerosas imágenes relacionadas con los conceptos de cada tema...

\section{Características de los libros}

Este periodo se corresponde con un boom editorial muy importante $y$, aunque nos hemos restringido a una muestra muy reducida de libros, hemos procurado que estuviera representada la mayor parte de las opciones existentes en el mercado. La estructura de los libros es típica de la enseñanza secundaria: están divididos en bloques de contenidos en los que se desarrollan diferentes temas según los conceptos a enseñar-aprender; se incorporan explicaciones detalladas, ejemplos relacionados con los conceptos, numerosos ejercicios resueltos y propuestos...

Hay una mayor cantidad de ejercicios y problemas, incrementándose respecto del periodo anterior. Además de los problemas clásicos que aparecen en todos los periodos, se incluyen algunos contextos novedosos como los relativos a contextos económicos y estadísticos, y se incorporan algunos ejercicios en los que la gran protagonista es la geometría cartesiana y los objetos de dicha geometría, utilizados bajo una formulación estrictamente algebraica. Por su peculiaridad destaca el libro del Grupo Cero, en el que la formulación y resolución de problemas es totalmente diferente del resto. Se busca más la compresión y la justificación de los conceptos que la aplicación rutinaria de ciertas reglas aprendidas de memoria.

En las definiciones se incorporan multitud de símbolos lógico-matemáticos, realizándose, en algunos casos, una traducción paralela entre el lenguaje habitual y el lógicomatemático, con lo que destaca su alto grado de abstracción. En cambio, en el libro del Grupo Cero, las definiciones se establecen a posteriori respecto de las explicaciones y ejemplos, y en el de Anaya del 88 se introducen algunos términos intuitivos. Los puntos críticos se definen como valores de las funciones que cumplen unas determinadas condiciones, resultando bastante similar al periodo anterior. Las tablas son muy numerosas, utilizándose exclusivamente para representar gráficamente funciones, aunque en el libro del Grupo Cero tienen entidad propia. Las gráficas son numerosas, incluyéndose no sólo los casos correspondientes a funciones derivables, sino también algunas que no lo son. Se utilizan diferentes tipos de gráficas aunque destacan por su importancia los mensajes topológicos que se mantienen desde su incorporación en la etapa anterior. En los libros del Grupo Cero y de Anaya 88, se incluyen numerosos ideogramas para la creación de una imagen visual, como medio de identificación de los conceptos. Entre las expresiones simbólicas, destacan las de tipo algebraico. En el libro del Grupo Cero se incluyen problemas en los que se relacionan las expresiones simbólicas con la descripción de algunas características gráficas de la función, con lo cual se intentan englobar varias representaciones en una misma actividad.

\section{Perfil}

Entre los libros que se han analizado correspondientes a este periodo, se ha elegido el libro del Grupo Cero (1982). Matemáticas de bachillerato. Volumen 2. Barcelona: Teide, por su carácter peculiar respecto del resto de los libros analizados de todos los periodos.

Durante esta época se produce una gran expansión editorial, consolidándose el paso de los libros de autor a los libros de editorial. Una notable excepción la constituye el libro publicado por el Grupo Cero de Valencia, que, siguiendo la fenomenología de Freudenthal, introduce y desarrolla los conceptos mediante una serie de actividades dirigidas.

Se encarga a un constructor unos bloques de viviendas y quiere, como es natural, minimizar el coste del apartado referente a ventanas, de tal manera que, manteniendo la misma $l u z$, la misma superficie de ventana, el coste del marco sea mínimo. He aquí cuatro ventanas:

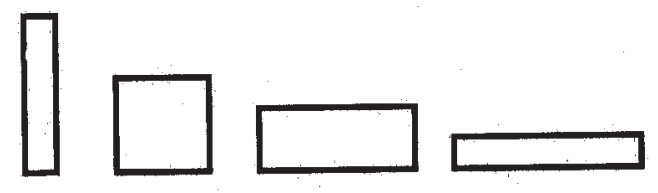

Todas las ventanas tienen la misma luz: $1 \mathrm{~m}^{2}$. Pero es evidente que el marco de la cuarta es más caro que el de las otras tres, porque tiene más perímetro.

¿Cuál es la ventana de marco más barato que tenga la misma $l u z, 1 \mathrm{~m}^{2}$ ? Lo que hay que estudiar es, por supuesto, el perímetro. Este perímetro es función de la altura de la ventana. (Grupo Cero, p. 102)

La estructura que posee este libro induce a pensar que el aprendizaje debe predominar sobre la enseñanza, ya que plantea situaciones en la que es el alumno el que tiene que investigar, conjeturar y rectificar, si es preciso, para alcanzar el conocimiento, siendo el papel del maestro el de gestor del aprendizaje. Los enunciados se expresan de forma explicativa, por lo que no poseen la estructura de los típicos problemas estándar. Así se puede deducir del siguiente problema. 
El departamento de marketing de una empresa estima que los ingresos mensuales que producirá el lanzamiento de un nuevo detergente vendrán dados, en miles de pesetas, por:

$$
y=200+6 t^{2}-\frac{t^{3}}{3}
$$

donde el tiempo $t$ viene expresado en meses a partir del lanzamiento al mercado del detergente. ¿Cuál es la tasa de variación instantánea de los ingresos a los cinco meses del lanzamiento?

¿Durante qué período de tiempo aumenta esta tasa?¿Durante qué periodo disminuye? ¿En qué momento es más alta? (Grupo Cero, p. 101)

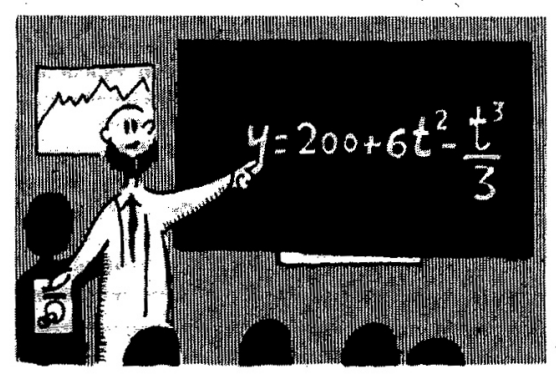

Por eso, el libro no señala un programa, sino que provee de materiales para el trabajo activo del alumno. En palabras de los autores, «en una clase activa de matemáticas, la tarea primordial es hacer matemáticas, es decir, matematizar», y entienden hacer matemáticas como la actividad intensa del alumno estudiando diversos fenómenos, con los conceptos matemáticos que sirven para organizarlos e interpretarlos. Se rompe la secuencia de los libros del bloque anterior: ejemplo - definición - propiedades - ejercicios, convirtiéndola en presentación de diversos fenómenosanálisis de estos fenómenos - introducción del concepto organizados - nuevos fenómenos - ejercicios.

Con estos planteamientos, y bajo la tutela del profesor, se pretenden desarrollar en los alumnos capacidades que no se habían considerado hasta entonces en los libros de texto españoles, como: inducir, conjeturar, experimentar, analizar, rectificar los propios errores, sintetizar... Para ello se utilizan en los enunciados de los problemas algunas referencias a la sociedad del momento: marcos de ventana, calderas, envases de cocina, depósitos para la tintorería.

En una tintorería necesitan depósitos para colocar el tinte, y para construirlos quieren aprovechar unas chapas rectangulares de $10 \mathrm{dm} \times 16$ $\mathrm{dm}$. Se construyen con estas chapas los depósitos por el procedimiento de cortar las esquinas de las planchas en la forma indicada en la figura, luego doblar y finalmente soldar.

a) Llamando $x$ a uno de los lados de cada uno de los cuadrados que se recortan, escribe la función $f: x \rightarrow V$ que da el volumen del depósito conocido $x$.

\section{b) ¿Para qué valor de $x$ se obtiene un depósito de volumen máximo?}

c) En el caso de que el volumen del depósito sea máximo, ¿cuántos kilos de tinte podrá contener cada uno de los depósitos si la densidad del tinte es de $1^{\prime} 20 \mathrm{~kg} / \mathrm{dm}^{3}$ ?(Grupo Cero, p. 108)
El tipo de situaciones, está equilibrado entre diferentes áreas, incluyendo contextos de tipo económico, cotidiano, y situaciones de tipo matemático. Entre estas últimas, también hay una gran diversidad, de forma que, además de contenidos aritméticos o geométricos, se incluyen otros. La mayoría de las interpretaciones son de tipo cualitativo, como analizar el tipo de envase que resulta según las dimensiones obtenidas... Y se puede observar un equilibrio entre los problemas de optimización y los ejercicios de representación gráfica de funciones.

Aunque la forma de presentación de los contenidos es estática, hay algunos atisbos de introducción de dinamismo. Por ejemplo, en el problema de los marcos de una ventana, se incluyen rectángulos con los que se quiere mostrar cómo varía el perímetro en función del área. Los problemas son fundamentalmente escolares, aunque en algunos los datos están adaptados a una hipotética situación real. A pesar de ello, no influyó mucho en los libros de la época, ni siquiera en los libros posteriores, en los que no se ha encontrado nada similar.

El uso de términos formales es más bien escaso, se utilizan en su lugar términos más intuitivos, tanto para introducir las definiciones como para enunciar las reglas, los teoremas y en el enunciado de los problemas.

\section{Intuitivamente:}

Un máximo es un punto que «está más alto» que sus vecinos.

Un mínimo es un punto que «está más bajo» que sus vecinos.

Con más precisión:

Una función tiene un máximo para $x=a$ si hay un intervalo que contenga a $a$ en el cual se cumpla que $f(a) \leq f(x)$ para todos los $x$ de ese intervalo. $f(a)$ es entonces el máximo.

Una función tiene un mínimo para $x=a$ si hay un intervalo que contenga a $a$ en el cual se cumpla que $f(a) \leq f(x)$ para todos los $x$ de ese intervalo. $f(a)$ es entonces el mínimo.

Fácilmente se comprende que no estamos refiriéndonos a máximo o mínimo globales (el mayor valor de la función o el menor valor de la función en todo su campo de existencia).

Decimos, pues, que la función tiene en ...MÁXIMOS LOCALES y en ... MÍNIMOS LOCALES.

Eventualmente, uno de los máximos locales puede ser máximo global ... Y uno de los mínimos locales puede ser un mínimo global. (Grupo Cero, p.101)

En las definiciones se incluyen ejemplos y contraejemplos que permiten interpretar las afirmaciones que se hacen a lo largo del texto.

Puede ocurrir que haya máximo o mínimo en $x=a$ y que no exista $f^{\prime}(a)$; un ejemplo es $f(x)=|x|$ en $\mathrm{x}=0 \quad$ (Grupo Cero, p. 105)

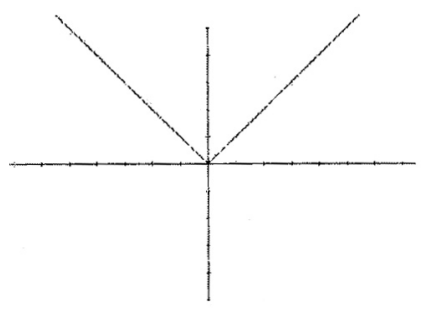


Hay un equilibrio en el tipo de funciones consideradas, de tal forma que prácticamente hay alguna de cada tipo, añadiéndose funciones definidas a trozos y otras en las que simplemente se da una descripción verbal de sus características. Se incluyen diferentes tipos de representación, de forma que se obtenga una imagen del concepto ajustada a sus diferentes posibilidades. Por ejemplo, en relación con la siguiente gráfica son los alumnos los que tienen que verbalizar las posiciones que ocupan algunos puntos notables de ella.

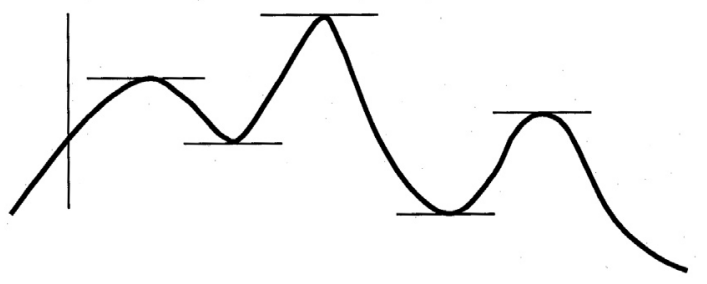

En la gráfica dada, ¿hay algún mínimo local que sea también mínimo global?

¿De qué modo las derivadas pueden ayudar a encontrar esos puntos? La respuesta es inmediata, porque, si se trazan las tangentes en ellos, esas tangentes son paralelas al eje de abscisas, luego tienen como pendiente 0. (Grupo Cero, p. 102)
Hay una gran utilización de herramientas de impresión, como el color, la diversidad de gráficos y tablas.

El libro del Grupo Cero es un libro de carácter comprensivo, aunque se ha procurado mantener el rigor en las definiciones, exponiéndolas como conclusión de ciertas observaciones realizadas a priori y no se olvida la práctica de las reglas necesarias para el cálculo de los puntos críticos con el fin de adquirir cierta destreza (Tabla V).

\subsection{Una nueva orientación de la enseñanza de las matemáticas bajo las orientaciones de la LOGSE: 1995-2001}

\section{Planes de estudio}

Este periodo comienza con la implantación de la Ley de Ordenación General del Sistema Educativo (LOGSE) en la educación secundaria de forma generalizada desde del año 1995 hasta la fecha. Aunque la Ley se promulgó en el año 1990 (3/10/1990), durante estos cinco años se fue implantando de forma progresiva en la enseñanza primaria y, de modo experimental, se impartieron anticipadamente los bachilleratos previstos en esta Ley.

Perfil del libro del Grupo Cero.

\begin{tabular}{|c|c|c|c|c|}
\hline Categorías & Dimensiones & Expositivo & Tecnológico & Comprensivo \\
\hline \multirow{5}{*}{ Sintáctica } & Estructura del problema & & & $*$ \\
\hline & Descripciones teóricas & & $*$ & $*$ \\
\hline & Símbolos utilizados en las tablas & & $*$ & \\
\hline & Símbolos utilizados en las gráficas & $*$ & & $*$ \\
\hline & Tipos de expresiones simbólicas & & * & \\
\hline \multirow{5}{*}{ Semántica } & Fenomenología & & & $*$ \\
\hline & Tipos de descripciones & & $*$ & $*$ \\
\hline & Tipos de tablas & & & $*$ \\
\hline & Tipos de gráficas & & & $*$ \\
\hline & Tipos de expresiones simbólicas & & $*$ & $*$ \\
\hline \multirow{5}{*}{$\begin{array}{l}\text { Pragmático- } \\
\text { didáctica }\end{array}$} & Función de los ejercicios & $*$ & $*$ & $*$ \\
\hline & Papel de las definiciones & & & $*$ \\
\hline & Actividades relacionadas con las tablas & & $*$ & $*$ \\
\hline & Actividades gráficas & $*$ & & $*$ \\
\hline & Papel de las expresiones simbólicas & $*$ & & \\
\hline \multirow{5}{*}{ Sociocultural } & Influencia social y adaptación al currículo & & & $*$ \\
\hline & Influencias didácticas & $*$ & & $*$ \\
\hline & Aplicación de las tablas & & & $*$ \\
\hline & Presentación de las gráficas & $*$ & & \\
\hline & Complejidad de las expresiones simbólicas & & * & \\
\hline
\end{tabular}


Se aumenta la enseñanza obligatoria hasta los 16 años (la enseñanza secundaria obligatoria 12-16 años) y el bachillerato se reduce de cuatro años a dos. Las Matemáticas no son una asignatura que estudien todos los alumnos de 16-18 años, son materia de modalidad sólo en el Bachillerato de Ciencias de la Naturaleza y de la Salud, Tecnología y Humanidades, y Ciencias Sociales, aunque con distinto carácter y distintos objetivos en cada una de ellas.

\section{Características de los libros}

En el periodo que nos ocupa se ha ido procediendo al desarrollo de la LOGSE, por lo que resulta difícil establecer unas características claramente identificativas de los libros escritos de acuerdo con esta Ley. Hemos de tener en cuenta además que hasta el año 2001 no ha sido generalizada la implantación en todos los institutos de enseñanza media de este plan de estudios. Pero podemos adelantar que, a pesar de las orientaciones establecidas en los programas oficiales, no han tenido un claro reflejo en el material curricular editado al efecto, de tal forma que la importancia concedida a la heurística, la resolución de problemas, la aplicación de las matemáticas a distintos ámbitos de la vida y el apoyo a la intuición, no se corresponden con las formulaciones que aparecen en los libros de texto analizados. El resultado ha sido la aparición de libros de texto muy voluminosos donde los conceptos están condensados en pocas palabras y en los que la actividad del alumno no se diferencia mucho de la llevada a cabo en periodos anteriores. La estructura de los libros es similar a la de los libros del periodo anterior, aunque hay una mayor profusión de imágenes, colores, recuadros, síntesis...

Los tipos de problemas y su cantidad han aumentado considerablemente. Así, además de los clásicos ya mencionados en otros periodos, tenemos: numerosos problemas que incluyen situaciones cotidianas, en los que se complican el tipo de formas geométricas a las que se hace referencia; son escasos, pero hay algún problema relacionado con la física; se mantienen los que se refieren a los contextos económicos y aumentan considerablemente los que se refieren a fenómenos matemáticos; se recuperan los problemas de tipo general que se describieron en el primer periodo y se introducen problemas de tipo gráfico, en los que hay que analizar la gráfica de una función o de su función derivada para calcular los puntos críticos. La definición que se establece de los puntos críticos se hace más bien a partir de la regla para calcularlos (anulación de la primera o de la segunda derivada) que a partir de una propiedad de la función o un comportamiento de la curva que la representa gráficamente. Se mantienen algunos símbolos lógico-matemáticos, pero se reduce considerablemente su uso. Hay numerosas tablas, pero siguen manteniendo una posición secundaria respecto del resto de las representaciones utilizadas. Se han modernizado, al igual que los libros, incorporando el color, y una maquetación de tipo más actual, pero su papel sigue siendo muy reducido. En cuanto a las gráficas, se introducen muchas características «escolares» como la utilización del color, de cuadrículas, flechas... Su papel no sólo corresponde a la visualización de las propiedades y a la construcción de imágenes visuales de los conceptos, sino que también se trata la clasificación de los puntos según sus posibilidades dentro de la gráfica. Se introducen algunas descripciones de funciones con el fin de averiguar su expresión simbólica, aunque la mayoría de los ejercicios son de tipo rutinario para encontrar los puntos extremos de una función normalmente polinómica. El único caso excepcional se refiere a una función determinada a partir de un determinante en el libro de Vizmanos. Aunque, en los periodos anteriores, las relaciones entre representaciones estaban relegadas a la traducción de una descripción a una expresión simbólica, de ésta a la tabular y finalmente a la gráfica, podemos considerar que algunas de las características expuestas respecto de este periodo nos hacen pensar que en estos libros se está intentando establecer otros tipos de traducciones entre representaciones, como pasar de la descripción a la gráfica directamente o de la gráfica a la expresión simbólica, pero este tipo de situaciones son todavía muy escasas y no están sistematizadas.

\section{Perfil}

El perfil que se incluye a continuación es el del libro de Primo, A. y otros (1998). Matemáticas (Modalidad: teconologías, ciencias de la naturaleza y la salud). Salamanca: Hespérides.

Este libro trata todos los conceptos pertenecientes al currículo, aunque se concede poca importancia a la intuición y desarrolla en cambio, una fuerte formalización en definiciones y teoremas. Se puede considerar que la adaptación al currículo es aceptable pero no hay ninguna influencia de las corrientes didácticas actuales, por lo que es de corte más bien tradicional. Entre los términos usados hay pocos de carácter intuitivo; en las definiciones se han incluido adverbios como mayor o menor pero, en general, hay una fuerte formalización.

Un punto $(c, f(c))$ de la gráfica de la función $f$ es un máximo local o relativo de $f$ si $f(c)$ es el mayor valor que toma $f$ en las proximidades de c, es decir, en algún entorno $I$ de $c$ contenido en su dominio de definición.

Un punto $(c, f(c))$ de la gráfica de una función $f$ es un mínimo local o relativo de $f$ si $f(c)$ es el menor valor que toma $f$ en un entorno de $c$ contenido en su dominio de definición.

Un máximo local (respectivamente mínimo local) $(c, f(c))$ se dice que es máximo absoluto (respectivamente mínimo absoluto) si $f(c)$ es el mayor valor (respectivamente el menor valor) o valor máximo (respectivamente valor mínimo) de $f$ en todo su dominio de definición. (Hespérides, p. 309)

Los problemas se resuelven siguiendo algunos de los pasos establecidos por Polya pero no se hacen interpretaciones de los resultados obtenidos ni se plantean problemas que supongan un verdadero reto a los alumnos. De hecho, la mayoría son problemas que han aparecido en los libros de texto a lo largo del resto de los periodos: 
Se dispone de $100 \mathrm{~m}$ de valla metálica para construir un cercado rectangular aprovechando una pared existente. Calcula las dimensiones de la cerca para que el área sea máxima. (Hespérides, p. 324)

La estructura de los enunciados de los problemas es totalmente rigurosa y clásica sin que haya lugar a la interpretación, la presentación de una situación desarrollada... Se incluyen contextos referidos a algunas áreas distintas de las matemáticas aunque, comparativamente, en menor número que aquéllos que se refieren exclusivamente a ámbitos propios de las matemáticas. Se hace referencia a contextos económicos, físicos o de naturaleza cotidiana.

Una empresa de ordenadores tiene unos ingresos y unos costes de producción que se ajustan a las siguientes funciones:

$$
\begin{aligned}
& I(x)=60 x-x^{2} \text { (función de ingresos) } \\
& x=\text { (número de unidades producidas) } \\
& C(x)=x^{2}-12 x+120 \text { (función de costes) }
\end{aligned}
$$

Se desea saber cuál es el beneficio máximo de la empresa y qué número de unidades es preciso producir para obtenerlo. (Hespérides, p. 319)

Dentro de las matemáticas prácticamente sólo se hace hincapié en los propios conceptos del análisis, aunque se integran otras áreas como aritmética, geometría euclídea, geometría cartesiana y trigonometría.

En el plano cartesiano se consideran tres puntos fijos $A_{1}, A_{2}$ y $A_{3}$ y una recta variable de dirección dada. Determina una recta de manera que la suma de los cuadrados de las distancias de estos tres puntos a ella sea mínima. Comprueba que la recta obtenida pasa por el punto $\mathrm{P},\left(\frac{x_{1}+x_{2}+x_{3}}{3}, \frac{y_{1}+y_{2}+y_{3}}{3}\right)$ siendo $A_{i}\left(x_{i}, y_{i}\right)$ las coordenadas de los puntos dados. (Hespérides, p. 324)

A pesar de ello, las soluciones que se obtienen en los problemas son de tipo algorítmico. Las presentaciones de conceptos, problemas, definiciones, etc. son totalmente estáticas, los problemas son de tipo escolar, y hemos de señalar, por ejemplo, lo inadecuado de algunas medidas (utilización de la peseta como unidad monetaria), la incorporación de algunas funciones de forma artificial en un contexto de tipo económico... No hay ni siquiera referencia a medios informáticos o de las nuevas tecnologías. La aplicación de los conceptos relativos a puntos extremos se lleva a cabo tanto en problemas de optimización como en ejercicios de representación gráfica de funciones.

Entre las expresiones utilizadas en relación con las funciones no hay, por ejemplo, funciones definidas a trozos u otras similares y sí se observa, sin embargo, una fuerte formalización en algunas expresiones algebraicas, siendo la más llamativa la que se refiere a las medidas de una cierta magnitud.

Si $x_{1}, x_{2} \ldots x_{n}$ son mediciones igualmente precisas de la magnitud $x$, su valor más probable será aquel para el que la suma de los cuadrados de los errores, $\sum_{i=1}^{n}\left(x-x_{\mathrm{i}}\right)^{2}$ tenga el valor mínimo ¿Cuál es el valor más probable? (Hespérides, p. 319)
Las tablas son de descripción local, en las que sólo se estudia el signo de la derivada primera que indica el crecimiento, decrecimiento y puntos críticos. En estas tablas aparecen además de símbolos numéricos, palabras de la lengua habitual relativas a las propiedades de las funciones como decrece, crece.

Hemos obtenido para la función $f(x)=x^{4}-4 x^{3}+4 x^{2}-2$ definida en $R$ los siguientes intervalos de crecimiento y decrecimiento:

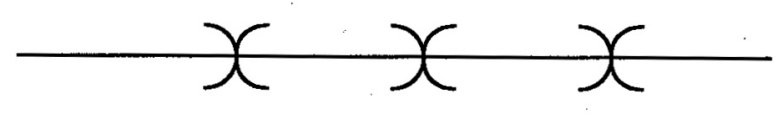

Decrece 0 Crece 1 Decrece 2 Crece

...lo que indica que en $x=0$ y $x=2$ la función presenta un mínimo local, y en $x=1$ un máximo local, esto es: $(0,-2)$ y $(2,-2)$ son los mínimos locales de $f$ y $(1,-1)$ es el máximo local de $f$. (Hespérides, p. 310)

Las gráficas son de tipo ábaco que se utilizan para estudiar el comportamiento gráfico de funciones particulares; es decir, a partir de una función se estudian sus máximos y mínimos, tanto absolutos como relativos. Solamente se señalan los valores en los que se anula la derivada, representando sus coordenadas, y asociando dichos puntos con su abscisa y ordenada correspondientes mediante líneas punteadas, que enfatizan el carácter de ábaco de dicha gráfica.

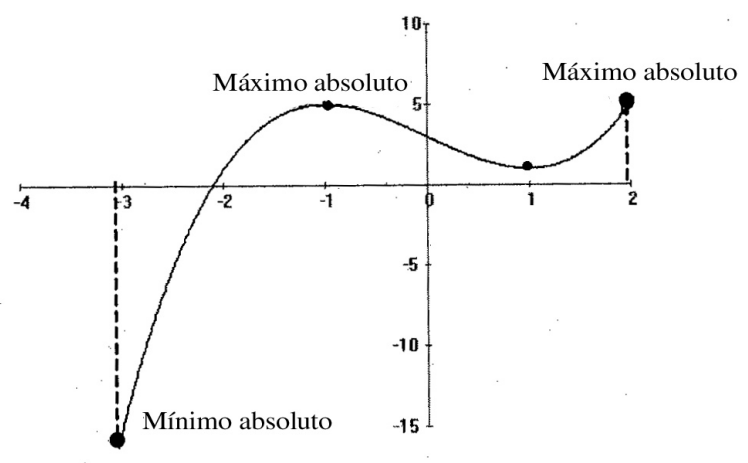

Prácticamente no hay interacción entre los distintos tipos de representaciones salvo algún esquema que se ha dibujado para representar el enunciado de algún problema.

El libro de Primo y otros es de carácter tecnológico, destaca la gran cantidad de ejercicios propuestos para practicar las reglas que se han expuesto, y para las que se ha utilizado una formulación de carácter abstracto-formal, que contribuye a forjar el carácter rígido y estático que posee este texto. El tipo de expresiones utilizadas no están enfocadas hacia la comprensión de los conceptos sino, como hemos dicho, hacia su utilización y por ello resulta realmente anacrónico el libro en relación con el enfoque de los planes oficiales (Tabla VI). 
Tabla VI

Perfil del libro de Primo y otros.

\begin{tabular}{|c|c|c|c|c|}
\hline Categorías & Dimensiones & Expositivo & Tecnológico & Comprensivo \\
\hline \multirow{5}{*}{ Sintáctica } & Estructura del problema & & $*$ & \\
\hline & Descripciones teóricas & & * & \\
\hline & Símbolos utilizados en las tablas & & $*$ & \\
\hline & Símbolos utilizados en las gráficas & $*$ & & \\
\hline & Tipos de expresiones simbólicas & & $*$ & \\
\hline \multirow{5}{*}{ Semántica } & Fenomenología & $*$ & $*$ & \\
\hline & Tipos de descripciones & & $*$ & \\
\hline & Tipos de tablas & & & * \\
\hline & Tipos de gráficas & & $*$ & * \\
\hline & Tipos de expresiones simbólicas & & * & * \\
\hline \multirow{5}{*}{$\begin{array}{l}\text { Pragmático- } \\
\text { didáctica }\end{array}$} & Función de los ejercicios & * & & \\
\hline & Papel de las definiciones & & $*$ & \\
\hline & Actividades relacionadas con las tablas & & $*$ & \\
\hline & Actividades gráficas & $*$ & & \\
\hline & Papel de las expresiones simbólicas & & $*$ & \\
\hline \multirow{5}{*}{ Sociocultural } & Influencia social y adaptación al currículo & $*$ & & \\
\hline & Influencias didácticas & & $*$ & \\
\hline & Aplicación de las tablas & & $*$ & \\
\hline & Presentación de las gráficas & $*$ & & \\
\hline & Complejidad de las expresiones simbólicas & & * & \\
\hline
\end{tabular}

\section{CONCLUSIONES}

A lo largo del siglo xx los libros de texto de educación secundaria en España han ido cambiando la forma de presentar los contenidos relativos a los puntos críticos tanto en relación con la formulación de las definiciones como en los tipos de problemas propuestos a los alumnos o en la forma en la que se han usado las gráficas en dichos libros. Estas variaciones se pueden caracterizar por medio del modelo de análisis de los libros de texto que hemos desarrollado, que además nos permite establecer un perfil que identifique dichos libros.

Si particularizamos en los diferentes elementos que hemos considerado en el análisis, debemos destacar que entre las definiciones que aparecen en los libros hemos encontrado tres tipos: funcionales, cuando se dice que, por ejemplo, el máximo es el mayor valor de la función; reglas, cuando se dice que su derivada ha de ser cero, o gráficas, cuando hace referencia a un punto, propiedad o comportamiento de una curva. De ellas, la mayoría de los libros identifican los puntos críticos con un punto de una curva, sin hacer referencia a la variación asociada a una función. Hemos de destacar además de la ausencia, en la definición de los puntos críticos, de la consideración de la posibilidad de tangente vertical. Esto contrasta con algunas investigaciones realizadas en relación con la posición de la tangente, en las que se consideran tanto los casos de puntos cuya tangente es horizontal como de aquéllos en los que es vertical.

En cuanto a los problemas planteados en relación con los puntos críticos, se ha producido un aumento progresivo en cada uno de los periodos al siguiente, tanto en el número de problemas como en el tipo de los problemas, destacando los problemas físicos en el primer periodo, los matemáticos en el segundo, los económicos en el tercero y los gráficos en el último. La mayoría son problemas numéricos y son escasos, salvo en algún periodo, los problemas más generales que conciernen a toda una familia de funciones. A pesar de las variaciones en cada uno de los libros, lo que no ha variado grandemente es el tipo de actividad que se espera del alumno, destacando la aplicación rutinaria de las reglas a ejercicios tipo o escolares.

En relación con las gráficas, destaca su papel en la visualización de los conceptos de punto crítico, pero también la clasificación de éstos. Por ello, el carácter de que se dota a los conceptos es fundamentalmente estático, al establecerse exclusivamente el resultado final. La utilización de las tablas ha sido muy escasa, siendo de apoyo a otro tipo de representaciones y, en cuanto a las expresiones simbólicas, se han considerado casi exclusivamente las de tipo algebraico, siendo escasas las funciones no 
derivables en los puntos críticos, así como cualquier tipo de función en el que su representación gráfica sea un poco más complicada de lo habitual.

En relación con los perfiles de los libros, hay que destacar que hay el mismo número de expositivos que de tecnológicos, sin que esto dependa del periodo al que corresponden. Esto hace pensar que el énfasis en los libros de texto está puesto, o bien en la exposición de los conceptos de una forma rigurosa, o bien en la adquisición de ciertas destrezas y habilidades calculísticas, sin que hayan influido claramente las nuevas corrientes en didáctica de las matemáticas sobre todo en los libros actuales. Destaca, en este sentido, el libro del Grupo Cero, por no adaptarse a las mismas características que el resto de los libros, aunque haya tenido una escasa influencia en el resto, dado que no hay ninguno escrito con posterioridad que se acerque a sus formulaciones.

De las consideraciones anteriores podemos también concluir que el tipo de orientación de los libros no depende de los planes de estudio en los que se encuadre, sobre todo en relación con los libros del último periodo, puesto que hemos visto que no se reflejan en las nuevas orientaciones dictadas por el Ministerio de Educación más que de una forma muy leve. Son los propios libros de texto los que establecen el tipo de actividad que debe ejercer el alumno y la forma en que se estructuran los conceptos matemáticos. Creemos, sin embargo, que el libro de texto debe ser sólo un material auxiliar de apoyo en la enseñanza, que se complemente tanto con otros libros de texto, libros de otro carácter como material diverso, didáctico o fungible, audiovisual u otro.

\section{NOTA}

Este artículo es parte de un proyecto de investigación financiado por la Junta de Castilla y León, por la Unión Europea (FSE) y por el Programa de Promoción General del Conocimiento de la DGES (Proyecto BX2000-0069). 


\section{REFERENCIAS BIBLIOGRÁFICAS}

CANTORAL, R. (1995). Los textos de cálculo: una visión de las reformas y contrarreformas. Documento inédito.

CHEVALLARD, Y. (1985). La transposition didactique du savoir savant au savoir enseigné. Grenoble: La Pensée Sauvage.

CHEVALLARD, Y. y JOSHUA, M.A. (1982). Un exemple d'analyse de la transposition didactique: La notion de distance. Recherche en Didactique des Mathématiques, 3(1), pp. 159-239.

CHOPPIN, A. (1980). L'histoire des manuels scolaires. Un bilan bibliométrique de la recherche français. Histoire de l'Education, 58, pp. 165-185.

DHOMBRES, J. (1984). French Mathematical Textbooks from Bézout to Cauchy. Historia Scientarum, 28, pp. 91-137.

DORMOLEN, J. VAN (1986). Textual Analisis, en Christiansen, B., Howson, A.G. y Otte, M. (eds.). Perspectives on Mathematics Education, pp. 141-171. Dordrecht: Reidel.

FILLOY, E. y ROJANO, T. (1984). From an Arithmetical to an Algebraic Thought (A clinical study with 12-13 years old), en Moser, J. (ed.). Proceedings on the Sixth Annual meeting for the Pshycology of Mathematics Education, North American Chapter, pp. 51-56. Madison, Wisconsin.

GARCÍA-RODEJA, I. (1997). ¿Qué propuestas de actividades hacen los libros de primaria? Alambique, 11, pp. 35-43.

GONZÁLEZ, M.T. (2002). «Sistemas simbólicos de representación en la enseñanza del análisis matemático: perspectiva histórica acerca de los puntos críticos». Tesis doctoral inédita. Universidad de Salamanca.

HOWSON, G. (1995). Mathematics Textbooks: A comparative Study of Grade 8 texts. Vancouver: Pacific Educational Press.

JANVIER, C. (ed.) (1987). Problems of Representation in the Teaching and Learning of Mathematics. Londres: Lawrence Earlbaum Associated Publishers.

LOWE, E. y PIMM, D. (1996). «This is so»: a text on texts, en Bishop, A., Calements, K., Keitel, C., Kilpatrick, J. y Laborde, C. International Handbook of Mathematics Education, pp. 371-410. Dordrecht: Kluwer.

MAZ, A. (2000). «Tratamiento de los números negativos en textos de matemáticas publicados en España en los siglos XVIII y XIX». Tesis de maestría. Granada: Universidad de Granada.

OTTE, M. (1986). What is a text?, en Christiansen, B., Howson, A.G., Otte, M. (eds.). Perspectives on mathematics education, pp. 173-203. Drodrecht: D. Reidel Publishing Company.
OTTE, M. (1997). What is a text?, en Christiansen, B., Howson, A.G., Otte, M. (eds). Perspectives on mathematics education, pp. 173-203. Dordrecht: D. Reidel Publishing Company.

PALAREA, M. (1999). La adquisición del lenguaje algebraico; reflexiones de una investigación. Uno, 40, pp. 3-28.

PEPIN, B. et al. (2001). Mathematics textbooks and their use in English, French and German classrooms: a way to understand teaching and learning culture. Zentralblatt für Didaktik der Mathematik, 33(5), pp. 158-175.

PIMM, D. (1987). Speaking mathematically. Nueva York: Routledge y Kegan Paul. Trad. cast. (1990). El lenguaje matemático en el aula. Madrid: Ministerio de Educación y Ciencia - Ediciones Morata.

PIMM, D. (1994). Mathematics classroom language form, function and force, en Bielher, R. Cholz, R.W., Sträßer, R. y Winkelmann, B. (eds.). Didactics of mathematics as a Scientific Discipline, pp. 159-169. Dordrecht: Kluwer.

PUIG, L. (1994). El De Numeris Datis de Jordanus Nemoratius como sistema matemático de signos. Mathesis, 10, pp. 47-92.

RADFORD, L. (1997). On Psychology, Historial Epistemology and the teaching of mathematics: Towards a Socio-cultural History of Mathematics. For the learning of mathematics, 17(1).

ROJANO, T. (1994). La matemática escolar como lenguaje. Nuevas perspectivas de investigación y enseñanza. Enseñanza de las Ciencias, 12(1), pp. 45-56.

SANZ, I. (1995). «La construcción del lenguaje matemático a través de libros escolares de matemáticas». Las configuraciones gráficas de datos. Tesis doctoral. Vizcaya: Universidad del País Vasco.

SIERRA, M., GONZÁLEZ, M.T. y LÓPEZ, C. (1999). Evolución histórica del concepto de límite funcional en los libros de texto de bachillerato y curso de orientación universitaria, 1940-1995. Enseñanza de las Ciencias, 17(3), pp. 463-476.

SIERRA, M., GONZÁLEZ, M.T. y LÓPEZ, C. (2003). El concepto de continuidad en los manuales escolares de educación secundaria de la segunda mitad del siglo xx. Educación Matemática, 15(1), pp. 21-49.

SCHUBRING, G. (1987). On the methodology of Analysing Historical Textbooks: Lacroix as Textbook Author. For the learning of mathematics, 7(3), pp. 41-51.

TIANA, A. (2000). El proyecto MANES y la investigación histórica sobre los manuales escolares (siglos XIX y XX) Historia de la Educación, 19, pp. 179-194. 


\section{ANEXO}

A continuación enumeramos las dimensiones que se han tenido en cuenta en relación con cada una de las categorías (síntáctica, semántica, pragmático-didáctica y sociocultural), estableciendo para cada uno de estas dimensiones las tres versiones diferentes que se pueden observar según que correspondan a una orientación expositiva (E), tecnológica $(\mathrm{T})$ o comprensiva $(\mathrm{C})$, correspondientes a cada uno de los tres tipos de libros que se han descrito anteriormente. Las dimensiones son las que se describen a continuación:

\section{SINTAXIS}

Dimensión 1: Estructura del problema

E1: Problemas clásicos en el sentido de que, a partir de un enunciado, se establece todo un conjunto de soluciones agrupadas por una característica común. Se trata de problemas de tipo teórico cuya estructura es de la forma: calcula.

T1: Problemas de aplicación en los que, a partir de un enunciado con datos numéricos, se hace una pregunta acerca de la solución. C1: Son problemas en los que se incluye una explicación a modo de justificación del sentido del problema en relación con el concepto que se está estudiando.

Dimensión 2: Descripciones teóricas

E2: Descripciones formales con algún elemento intuitivo.

T2: Descripciones teóricas con símbolos lógico-matemáticos.

C2: Descripciones explicativas.

Dimensión 3: Símbolos utilizados en las tablas

E3: No se incluyen tablas.

T3: En las tablas se incluyen símbolos exclusivamente matemáticos.

C3: Se introducen en las tablas iconos como flechas, arcos...

Dimensión 4: Símbolos utilizados en las gráficas

E4: Gráficas generales en las que los datos se incluyen en forma literal.

T4: En las gráficas se incluyen símbolos numéricos.

C4: No hay ningún símbolo además de la propia gráfica.

Dimensión 5: Tipos de expresiones simbólicas

E5: Las expresiones simbólicas se refieren a familias de funciones.

T5: Expresiones simbólicas específicas.

C5: Expresiones simbólicas no habituales.

\section{SEMÁNTICA}

Dimensión 6: Fenomenología

E6: Problemas matemáticos.

T6: Problemas realistas.

C6: Problemas sacados de la realidad.

Dimensión 7: Tipos de descripciones

E7: Descripciones de los conceptos como una propiedad de las funciones.

T7: Descripciones en las que se enfatiza su aplicación: reglas $\left(f(x)>f\left(x_{0}\right)\right.$ o $\left.f^{\prime}(x)=0\right)$.

C7: Descripciones como propiedades de las curvas.

Dimensión 8: Tipos de tablas

E8: Ausencia de tablas.

T8: Cuadros variacionales en los que se enfatizan las relaciones o conexiones.

C8: Tablas de descripción local.

Dimensión 9: Tipos de gráficas

E9: Mensajes topológicos.

T9: Ábacos

C9: Gráficas como ideogramas.

Dimensión 10: Significado de las expresiones simbólicas

E10: Expresiones simbólicas como objetos manipulables.

T10: Expresiones simbólicas como iconos.

C10: Funciones en las que se refleja un proceso variacional. 


\section{PRAGMÁTICO-DIDÁCTICA}

Dimensión 11: Función de los ejercicios

E11: Ejercicios resueltos de aplicación de conceptos sin gráficos.

T11: Ejercicios propuestos de aplicación de reglas o ejercicios con apoyos gráficos o fórmulas.

C11: Problemas para la construcción de conceptos y reglas o con gráficos explicativos.

Dimensión 12: Papel de las definiciones

E12: Descripciones para la construcción de la teoría formuladas a priori.

T12: Desarrollo de reglas algorítmicas para la aplicación a problemas y ejercicios: énfasis técnicos.

C12: Interpretación de las reglas a partir de los fenómenos estudiados, estableciendo las definiciones a posteriori.

Dimensión 13: Actividades relacionadas con las tablas

E13: Ausencia de tablas

T13: Tablas construidas a partir de una expresión simbólica.

C13: Tablas para completar relacionadas en ambas direcciones con otras representaciones tanto para su construcción como para su interpretación.

Dimensión 14: Actividades gráficas

E14: Clasificación de conceptos.

T14: Construcción de gráficas.

C14: Visualización de propiedades.

Dimensión 15: Papel de las expresiones simbólicas

E15: Expresiones simbólicas utilizadas como ejemplos de los conceptos.

T15: Expresiones simbólicas para la aplicación de las reglas.

C15: Expresiones simbólicas que permitan establecer relaciones con otras formas de representación.

\section{SOCIOCULTURAL}

Dimensión 16: Influencia social y adaptación al currículum

E16: Sin contexto.

T16: Utilización de contextos atemporales.

C16: Contextos con referencias a la época en que fue escrito el libro.

Dimensión 17: Influencias didácticas

E17: Definiciones que mantienen la estructura de los libros históricos.

T17: Definiciones adaptadas a los planes de estudio y las orientaciones oficiales.

C17: Definiciones influenciadas por novedosas corrientes didácticas.

Dimensión 18: Aplicación de las tablas

E18: Ausencia de tablas.

T18: Tablas como elemento auxiliar.

C18: Tablas con categoría como cualquier otra representación.

Dimensión 19: Presentación de las gráficas

E19: Gráficas cartesianas sin añadidos.

T19: Gráficas con algún elemento característico del periodo (intervalos...).

C19: Utilización de gráficas con elementos escolares (cuadrículas, pantallas...).

Dimensión 20: Complejidad de las expresiones simbólicas

E20: Expresiones simbólicas clásicas representativas de ciertas curvas geométricas como la circunferencia, cicloide...

T20: Expresiones simbólicas escogidas porque su estructura es adecuada como aplicación didáctica de las reglas establecidas previamente.

C20: Expresiones muy variadas y complejas que puedan estar relacionadas con fenómenos reales y requieran el uso de las nuevas tecnologías. 